\title{
Trends in Incidence of Common Cancers in Iran
}

\author{
Mostafa Enayatrad ${ }^{1}$, Maryam Mirzaei ${ }^{2}$, Hamid Salehiniya ${ }^{3,4 *}$, Mohammad Reza \\ Karimirad $^{5}$, Siavash Vaziri ${ }^{6}$, Fiezollah Mansouri ${ }^{6}$, Asieh Moudi ${ }^{7}$
}

\begin{abstract}
Cancer is a major public health problem in Iran. The aim of this study was to evaluate trends in incidence of ten common cancers in Iran, based on the national cancer registry reports from 2004 to 2009. This epidemiological study was carried out based on existing age-standardized estimate cancer data from the national report on cancer registry/Ministry of Health in Iran. The obtained data were analyzed by test for linear trend and $\mathrm{P} \geq 0.05$ was taken as the significant level. Totals of 41,169 and 32,898 cases of cancer were registered in men and females, respectively, during these years. Overall age-standard incidence rates (ASRs) per 100,000 population according to primary site weres 125.6 and 113.4 in males and females, respectively. Between 2004 and 2009, the ten most common cancers (excluding skin cancer) were stomach (16.2), bladder (12.6), prostate (11), colon-rectum (10.14), hematopoeitic system (7.1), lung (6.1), esophagus (6.4), brain (3.2), lymph node (3.8) and larynx (3.4) in males; and in females were breast (27.4), colon-rectum (9.3), stomach (7.6), esophagus (6.4), hematopoeitic system (4.9), thyroid (3.9), ovary (3.6), corpus uteri (2.9), bladder (3.2) and lung (2.6). Moreover, results showed that skin cancer was estimated as the most common cancer in both sexes. The lowest and the highest incidence in females and males were reported respectively in 2004 and 2009. Over this period, the incidence of cancer in both sexes has been significantly increasing $(p<0.01)$. Like other less developed and epidemiologically transitioning countries, the trend of age-standardized incidence rate of cancer in Iran is rising. Due to the increasing trends, the future burden of cancer in the Iran is going to be acute with the expected increases in aging populations. Determining and controlling potential risk factors of cancer should hopefully lead to decrease in its burden.
\end{abstract}

Keywords: Cancer incidence - population - based cancer registry - trends - Iran

Asian Pac J Cancer Prev, 17, Cancer Control in Western Asia Special Issue, 39-42

\section{Introduction}

Cancer is a worldwide problem; millions of patients are diagnosed with different types of cancer throughout the world. More than half of the patients with cancer in the world are in developing countries (Ferlay et al., 2013 Apr). The incidence of cancer is rising in developed countries (Parkin, 2001). Based on the World Health Organization (W.H.O) report, it is estimated that the death rate due to cancer increases from 80 to $100 \%$ in the Middle East in the next 15 years (Ferlay et al., 2015; Omar et al., 2007). About 7.6 million cancer deaths occurred throughout the world in 2008, and about $60 \%$ of cancer deaths occur in low-income and middle-income countries and this number is prone to increase in the future. It is estimated that more than a quarter of a million people will die yearly in the Eastern Mediterranean Region (EMRO) alone. Iran is a member of the EMRO. The most common cancers in the EMRO are breast cancer in females, and lung and bladder cancers in males ((WHO),; Rafiemanesh et al., 2015a). Age specific incidence rate for all cancer in world is 205.4 (per 100,000 population) and mortality rate is 126.3 per 100,000 population in 2012 ; this rate is respectively 174.1 and 126.3 in Asia and is 134.7 and 90.4 in Iran (Ferlay et al., 2013 Apr). According to the report of cancer registry center, ten most common cancers in the country (Iran) are skin, stomach, breast, colorectal, bladder, esophagus, hematopoietic glands, prostate, lymph nodes, and lung, respectively (Goya, 2007.), mainly due to changes in lifestyle and nutrition in different countries, increasing mean age, and life expectancy. Cancer incidence has increased in any population. It is predicted that cancer will be the first and most important cause of death by 2030 (Karami et al., 2014; Rafiemanesh et al., 2015b; Razi et al., 2015b).

Cancers are the third cause of death in Iran after

${ }^{1}$ Department of Epidemiology, School of Public Health, Shahid Beheshti University of Medical Sciences, ${ }^{2}$ Tabriz University of Medical Sciences, Tabriz, ${ }^{3}$ Zabol University of Medical Sciences,Zabol, ${ }^{4}$ Department of Epidemiology and Biostatistics, School of Public Health, Tehran University of Medical Sciences, Tehran, ${ }^{5}$ Hormozgan University of Medical Sciences, Bandar Abbas, ${ }^{6}$ Department of Infectious and Tropical Disease, School of Medicine, Kermanshah University of Medical Sciences, Kermanshah, ${ }^{7}$ Birjand University of Medical Sciences, Birjand, Iran.*For correspondance: alesaleh70@yahoo.com 
cardiovascular diseases and accidents according to research performed in the country (Mousavi et al., 2009; Mirzaei et al., 2015; Razi et al., 2015b). A difference in cancer incidence changes is remarkable in the country, which is consistent with global patterns. Hence, planning for prevention and control of the disease is medical necessity and requires investigating trend of cancer in the past (Amoori et al., 2014; Karami et al., 2014; Rafiemanesh et al., 2015b; Razi et al., 2015a; Zahedi et al., 2015). Due to many reasons, including increasing elderly population, change in lifestyle and enhancing cancer-causing behaviors such as increasing tobacco consumption, unhealthy diet, lack of physical activity and other factors, the global burden of cancer is increasing (Moradpour and Fatemi, 2013). Therefore, national programs are essential for prevention and control of cancer in any society. Cancer registry is considered as a main tool for management and control of the disease (Goya, 2007). In population based cancer registries, there is data collected from cancer incidence and mortality from the disease obtained from a target group to describe epidemiology of cancer in a defined region, plan cancer control strategies, conduct, evaluate cancer prevention and control effects by scientific research. Cancer statistics is widely used in scientific studies, clinical trials, and making decisions on cancer prevention and control (Chen et al., 2013).

The primary purposes of cancer control programs are early detection, effective treatment, and palliative care programs, in which target population is not limited to certain age, sex, ethnicity, and race (Goya, 2007; Zahedi et al., 2015). Knowing the statistics of cancer and its trend is effective. This study aimed to investigate common cancer incidence and trend of its changes in Iran.

\section{Material and Methods}

This analytic study was done based on longitudinal national registry of cancer (NCR) program in Iran. Data used in this study was obtained from NCR, and Disease Control and Prevention (CDC) report of Iran Ministry of Health and Medical Education from 2004 to 2009 in Iran (Goya, 2007) .

Cancer registry system has been designed and implemented in Iran since 1986 and the first report was published in 1986. Data were collected retrospectively reviewing all new cancer patients registered in Cancer Registry Center of Health Deputy for Iran during a 6-year period (2004 - 2009). Data included demographic characteristics like age, sex residency, and date of diagnosis which were coded based on International Classification of Diseases for Oncology (ICD-O)

The number of cases, and crude and standardized incidence rates were also examined by sex and each province. Data were analyzed using Cochran - Armitage test for linear trend and software of Win Pepi 2.1 .

\section{Results}

Based on the national report on cancer registry, 74,067
Table1. Most Common Malignancies in Female

\begin{tabular}{lccccc}
\hline type of cancer & 2009 & 2007 & 2006 & 2005 & 2004 \\
\hline Breast & 28.2 & 33.2 & 27.1 & 25.1 & 23.2 \\
skin & 13.1 & 15.8 & 14.5 & 13.8 & 13.2 \\
colon & 10.9 & 11.1 & 8.8 & 8.2 & 7.4 \\
stomach & 7.8 & 10.0 & 7.4 & 6.6 & 6.1 \\
Esophagus & 5.8 & 7.8 & 6.1 & 6.1 & 6.1 \\
Hemato.sys & - & 6.3 & 4.6 & 4.4 & 3.7 \\
thyroid & 4.2 & 4.5 & 3.4 & 3.8 & 3.5 \\
ovary & 4.0 & 4.2 & 3.7 & 3.2 & 2.9 \\
Corpus uteri & 2.8 & 3.9 & 2.9 & 2.6 & 2.4 \\
bladder & 3.3 & 3.8 & 3.3 & 2.9 & 2.9 \\
Lung & 3.4 & 3.5 & 2.3 & 1.9 & 2.0 \\
\hline
\end{tabular}

cases of cancer had been recorded between 2003 and 2009, among which 32,898 and 41,169 cases were females and males, respectively. Overall age-standard incidence rate (ASR), per 100,000 individuals according to primary site is 125.6 and 113.46 in males and females, respectively. In this study, all cases of ten common cancers were examined based on cancer registry data. Between 2004 and 2009 years, the ASRs per 100,000 individuals, for ten most common cancers (excluding skin cancer) are stomach (16.2), bladder (12.6), prostate (11.0), colon-rectum (10.1), Hemeto.sys (7.1), lung (6.1), esophagus (6.4), brain (3.2), Lymph node (3.8) and larynx (3.4), in males; and in females are breast (27.4), colon-rectum (9.3), stomach (7.6), esophagus (6.4), Hemeto.sys (4.8), thyroid (3.9), ovary (3.6), Corpus uteri (2.9), bladder (3.2) and lung (2.6). Moreover, results showed that skin cancer was estimated as the one common cancer in both sexes. The lowest and the highest incidence in females and males have been reported respectively in 2004 and 2009 (Table 1

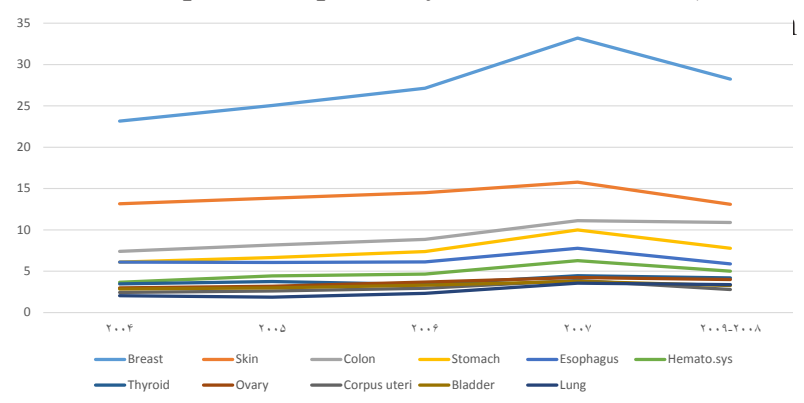

Figure 1. Trends in Most Common Cancer Incidence: Females Age Standardized Rate (ASR)

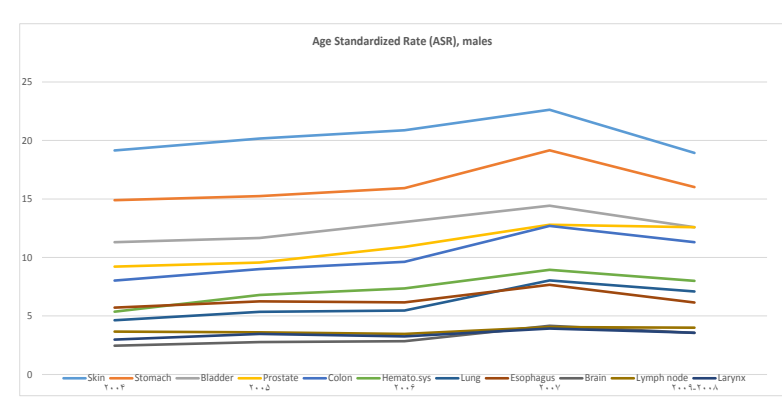

Figure 2: Trends in Most Common Cancer Incidence: Male Age Standardized Rate (ASR) 
Table2. Most Common Malignancies in Male

\begin{tabular}{lccccc}
\hline type of cancer & 2009 & 2007 & 2006 & 2005 & 2004 \\
\hline skin & 18.9 & 22.6 & 20.9 & 20.2 & 19.1 \\
stomach & 16.0 & 19.2 & 15.9 & 15.2 & 14.9 \\
bladder & 12.6 & 14.4 & 13.0 & 11.7 & 11.3 \\
prostate & 12.6 & 12.8 & 10.9 & 9.6 & 9.2 \\
colon & 11.3 & 12.7 & 9.6 & 9.0 & 8.0 \\
Hemato.sys & - & 8.9 & 7.4 & 6.8 & 5.4 \\
lung & 7.1 & 8.0 & 5.5 & 5.3 & 4.6 \\
esophagus & 6.1 & 7.7 & 6.2 & 6.2 & 5.7 \\
brain & 3.5 & 4.2 & 2.8 & 2.8 & 2.5 \\
Lymph node & - & 4.05 & 3.47 & 3.6 & 3.7 \\
Larynx & 3.6 & 3.9 & 3.3 & 3.5 & 3.0 \\
\hline
\end{tabular}

cancers have an increasing trend over time (Figure 1 and 2). Over this period, the Cochran - Armitage test indicated

that a significant trend was seen in the incidence of common cancer in both sexes during years studied (chi2, $\mathrm{p} \leq 0.01)$.

\section{Discussion}

According to our data, between 2003 and 2009, the age-standardized incidence rate of the cancer has increased in Iran. There was an increasing and significant trend. Our findings showed that cancer incidence is more in males than females; ASR was 125.6 in men and 113.4 in per 100,000 women. Based on the national report of cancer registry in 2009, ASR was 132.2 in men and 123.5 in women. The incidence was 132.6 and 133 in Pakistan in men and women, respectively (Bhurgri and Hassan, 2002). In Belgium, the cancer incidence in men and women were 400 and 322 per 100,000 people; in Canada, in men and women they were 158.2 and 170.8; in Lebanon, in men and women they were 141.4 and 126.8; in Europe, men and women they were 446 and in the world, the cancer incidence in men and women were 303 and 204 per 100,000 people respectively (Asulin et al., 2004). Therefore, the incidence of cancer in Iran was lower than some areas in the word. It seems that the cancer incidence estimated in Iran was not real and we underestimate the incidence of cancer in Iran, due to problems such as low quality of cancer registry system in Iran and the fact that in many cases we do not perform biopsy and therefore the disease would go undiagnosed in this case. In contrast, in certain developed countries in the last decade, the incidence and mortality of cancers had decreasing trends (Jemal et al., 2010; Bray et al., 2012). This difference might be due to fast changes in lifestyle, exposure to risk factors, aggregation of carcinogens, and air and environmental pollution in Iran that led to increasing trend in cancer incidence.

In our study, the most common cancer in women were breast, colorectal, stomach and esophagus, hematopoietic system, thyroid, ovarian, cervical, bladder and lung, respectively. While in men the most common cancers included stomach, bladder, prostate, colorectal, hematopoietic system, lung, esophagus, brain, lymph nodes, and Larynx respectively. Also during the years under review, skin cancer has been one of the most common cancers in both sexes. Five common cancers in men in the world are: lung, prostate, colorectal, stomach, and liver and in women, are breast, colorectal, lung, cervical, and stomach (World.Health.Organization, 2012). In the country (Iran), five common cancers in men are skin, stomach, prostate, bladder, and colorectal cancer, while in women, breast, skin, colorectal, stomach, and esophagus (Mohotrio, Summer 2011). Nevertheless, cancer incidence is different in various regions but ten common cancers in men in world are lung, prostate, pancreatic, lymph nodes, hematopoietic system, esophagus, stomach, bladder, kidney, and pharynx, respectively. Ten most common cancers in women are lung, breast, pancreatic, lymph nodes, hematopoietic system, stomach, cervix, kidney, rectum, and bladder, respectively (Ferlay et al., 2015; Keyghobadi et al., 2015; Koohi et al., 2015; Rafiemanesh et al., 2015b; Razi et al., 2015b).

There was a small difference between our results and national reports, but thee was a considerable difference between them and world reports. The differences in the incidence of cancer should be investigated to perform identifying related risk factors. Other studies also expressed high incidence of stomach cancer in men and breast cancer in women.

In conclusion, although the incidence of cancer has increased in Iran, the incidence of cancer in Western countries is less developed. According to changing lifestyles and dietary habits, it is possible that in the coming years we will be faced with a higher incidence of cancer in the country; Therefore, it is recommended to promote the use of screening programs and to increase awareness of the population is somewhat reduced the incidence of cancer in the country.

\section{References}

Amoori N, Mirzaei M, Cheraghi M(2014). Incidence of Cancers in Kuzestan Province of Iran: Trend from 2004 to 2008. Asian Pac J Cancer Prev 2005, 15, 8345-9.

Asulin Y, McCann T, MaCarty C, et al (2004). Cancer incidence and mortality in Grenada 1990-2000. West Indian Med J, 6, 368-73.

Bhurgri YBA, Hassan S (2002). Cancer pattern in Karachi Division (1998-1999). J Pak Med Assoc, 52, 244-6.

Bray F, Jemal A, Grey N, et al (2012). Global cancer transitions according to the Human Development Index (2008-2030): a population-based study. Lancet Oncol, 13, 790-801.

Chen WZ, Zhang S, Zhao P, et al (2013). The incidences and mortalities of major cancers in China, 2009. Chinese $J$ Cancer, 32, 106-12.

Ferlay J, Soerjomataram I, Dikshit R, et al (1 March 2015). Cancer incidence and mortality worldwide: Sources, methods and major patterns in GLOBOCAN 2012. Int $J$ Cancer, 136, E359-E86.

Ferlay J, Steliarova-Foucher E, Lortet-Tieulent J, et al (2013 Apr). Cancer incidence and mortality patterns in Europe: estimates for 40 countries in 2012. Eur J Cancer, 49, 1374-403.

Goya M (2007). Iranian annual cancer registration report 2005/2006. , Tehran, Iran., ministry of health and medical education, health deputy. center for disease control and 

prevention 2007. InPersian.

Goya M, (2007). Iranian Annual Cancer Registration Report 2003/2008., Tehran, Ministry of Health and Medical Education, Health Deputy. Center for Disease Control and Prevention.

Jemal A, Center MM, DeSantis C, et al (2010). Global patterns of cancer incidence and mortality rates and trends. Cancer Epidemiol Biomarkers Prev, 19, 1893-907.

Karami K, Cheraghi M, Amori N, et al (2014). Common Cancers in Khuzestan Province, South West of Iran, during 2005-2011. Asian Pac J Cancer Prev, 15, 9475-8.

Keyghobadi N, Rafiemanesh H, Hafshejani AM, et al (2015). Epidemiology and Trend of Cancers in the Province of Kerman: Southeast of Iran. Asian Pac J Cancer Prev, 16, 1409-13.

Koohi F, Enayatrad M, Salehiniya H (2015). A Study of the Epidemiology and Trends in Cancer Incidence in Iranian Elderly 2003-2009. J Arak Univ Med Sci, 18, 57-66.

Mirzaei M, Pournamdar Z, Salehiniya H (2015). Epidemiology and trends in incidence of kidney cancer in Iran. Asian Pac J Cancer Prev, 16, 5859-61.

Moradpour F, Fatemi Z (2013). Estimation of the projections of the incidence rates, mortality and prevalence due to common cancer site in Isfahan, Iran. . Asian Pac J Cancer Prev, 14, 3581-5.

Mousavi S, Gouya M, Ramazani R, et al (2009). Cancer incidence and mortality in Iran. Ann Oncol, 20, 556-63.

Omar S, Nelly H. Alieldin, Khatib OM (2007 Nov-Dec). cancer magnitude, challenges and control in the Eastern Mediterranean Region. East Mediterr Health J, 13, 1486-96.

Parkin DM (2001 Sep). Global cancer statistics in the year 2000. Lancet Oncol, 2, 533-43.

Rafiemanesh H, Mehtarpoor M, Mohammadian -Hafshejani A, et al (2015a). Cancer epidemiology and trends in Sistan and Baluchestan province, Iran. Med J Islam Repub Iran, 29, 1-7.

Rafiemanesh H, Rajaei-Behbahani N, Khani Y, et al (2015b). Incidence trend and epidemiology of common cancers in the center of Iran. Glob J Health Sci, 8, 146-55.

Razi S, Enayatrad M, Mohammadian-Hafshejani a, et al (2015a). The epidemiology of skin cancer and its trend in Iran. Int $J$ Prev Med, 6, 221-9.

Razi S, salehiniya H, Fathali-loy-dizaji M(2015b). Epidemiology of prevalent cancer among Iranian women and its incidence trends from 2003-2009 in Iran. J Arak Univ Med Sci, 18, $17-24$.

WHO (2014). [Accessed http://wwwemrowhoint/health-topics/ cancer/indexhtml.

World.Health.Organization (2012). Globocan Estimation Cancer Incidence, Mortality and Prevalence Worldwide in 2012, International Agency for Research on Cancer.

Zahedi A, Rafiemanesh H, Enayatrad M, et al (2015). Incidence, Trends and Epidemiology of Cancers in North West of Iran. Asian Pac J Cancer Prev, 16, 7189-93. 